\title{
MATERIAIS HÍBRIDOS ORGÂNICO-INORGÂNICOS: PREPARAÇÃo E ALGUMAS APLICAÇÕES
}

\author{
Nadia Mamede José*
}

Departamento de Físico Química, Instituto de Química, Universidade Federal da Bahia, Campus de Ondina, 40170-290 Salvador - BA Luís Antônio Sanchez de Almeida Prado

Technische Universität Hamburg-Harburg, Hamburg, Deutschland D-21073

Recebido em 29/1/04; aceito em 19/7/04; publicado na web em 5/11/04

\begin{abstract}
HYBRID ORGANIC-INORGANIC MATERIALS: PREPARATION AND SOME APPLICATIONS. The preparation and application of organic-inorganic hybrid materials are under fast development and constitute an interesting research topic on account of the versatility and wide range of applications offered by these materials. These properties can be achieved due to the mixture of the components at the molecular level. The present review covers the state of the art, the most useful preparation routes and the potential applications of these materials.
\end{abstract}

Keywords: organic-inorganic hybrid materials; structure-properties-applicability relationship; materials science.

\section{INTRODUÇÃO}

As mais recentes tecnologias requerem materiais com combinação de propriedades que não são encontradas nos materiais convencionais. Materiais híbridos orgânico-inorgânicos são preparados pela combinação de componentes orgânicos e inorgânicos e constituem uma alternativa para a produção de novos materiais multifuncionais, com uma larga faixa de aplicações. Normalmente as características desejadas não são encontradas em um único constituinte e a combinação adequada dos componentes tem levado à formação de materiais que apresentam propriedades complementares, que não são encontradas em uma única substância.

Híbridos orgânico-inorgânicos são materiais de grande interesse em aplicações comerciais devido às suas propriedades mecânicas, ópticas e térmicas, que combinam a estabilidade térmica e química dos materiais cerâmicos, com a processabilidade e a flexibilidade dos compostos e polímeros orgânicos. O desenvolvimento desta área acelerou-se desde a década de 80 , destacando-se a preparação de géis inorgânicos, impregnados por polímeros orgânicos ${ }^{1}$.

De um modo geral, esses materiais podem ser preparados de três modos: pela incorporação apenas física dos constituintes, através de ligações químicas entre os componentes e ainda uma terceira classe de materiais híbridos, baseada na combinação dos dois tipos de interação descritos acima. No segundo caso, destacam-se os híbridos que apresentam ligações covalentes entre o componente orgânico e o inorgânico.

Uma das principais rotas de obtenção de materiais híbridos orgânico-inorgânicos é o processo sol-gel. Os géis poliméricos são, geralmente, preparados a partir de soluções, onde promovem-se as reações de hidrólise e condensação. Outras técnicas de preparação e aplicações desses materiais serão apresentadas neste trabalho.

Em vista do grande potencial e caráter multidisciplinar, pesquisas voltadas a esta área vêm aumentando nos últimos anos e atraindo a atenção, tanto de pesquisadores acadêmicos, de diferentes áreas, como despertando o interesse para uso industrial. Este trabalho tem por objetivo demonstrar a potencialidade e o estado da arte de materiais híbridos orgânico-inorgânicos e discutir as principais publicações relacionadas à preparação e utilização desses materiais.

*e-mail: nadia@ufba.br

\section{DEFINIÇÃo}

Um dos mais antigos materiais constituídos de componentes orgânicos e inorgânicos provém da indústria de tintas, onde se adicionavam pigmentos inorgânicos em misturas orgânicas, embora nessa época o termo "híbrido orgânico-inorgânico" ainda não fosse mencionado $^{2}$. Esse termo começou a ser usado muito recentemente, nos últimos 20 anos, com o desenvolvimento de ORMOSILS, silicatos organicamente modificados ${ }^{3}$.

Materiais híbridos orgânico-inorgânicos são constituídos pela combinação dos componentes orgânicos e inorgânicos que, normalmente, apresentam propriedades complementares, dando origem a um único material com propriedades diferenciadas daquelas que lhe deram origem. Esses materiais são homogêneos, devido à mistura dos componentes em nível molecular, usualmente em escala de nanômetro a sub-micrômetro ${ }^{4}$. Embora tais materiais sejam macroscopicamente homogêneos, suas propriedades refletem a natureza química dos blocos pelos quais foram formados ${ }^{4,5}$.

Os termos compósitos e híbridos orgânico-inorgânicos têm sido, inadvertidamente, empregados na literatura como sinônimos. Compósitos são materiais constituídos por uma mistura de dois ou mais componentes ou fases distintas ${ }^{6}$, usualmente combinados em escala macroscópica, os quais devem estar presentes em proporções razoáveis ( $>5 \%)$. Deste modo, compósito e híbrido diferem entre si nas dimensões e na dispersão de seus componentes ${ }^{4}$; além disso, um compósito não é, necessariamente, constituído por componentes orgânicos e inorgânicos. O termo nanocompósitos foi introduzido por Roy e colaboradores ${ }^{7}$, entre 1982-1983, para designar materiais compósitos contendo mais de uma fase sólida, nos quais a fase dispersa apresenta, pelo menos, uma das suas dimensões em escala nanométrica, tratando-se, por conseguinte, de um tipo particular de compósitos $^{8}$. Os nanocompósitos podem ser divididos em três tipos, em função das dimensões da fase dispersa: isodimensionais, quando as três dimensões são da ordem de nanômetros (nanopartículas esféricas de sílica), no segundo tipo, duas dimensões estão em escala nanométrica (nanotubos de carbono ou "whiskers" de celulose) e o terceiro tipo, quando apenas uma dimensão da fase dispersa está em escala nanométrica, sob a forma de lâminas (materiais lamelares) ${ }^{8}$.

As propriedades de um material híbrido não são apenas a soma das contribuições individuais de seus constituintes; existe um 
sinergismo que depende também da natureza química dos segmentos orgânicos e inorgânicos e do tamanho e morfologia dos correspondentes domínios.

Como as fases têm dimensões moleculares, a influência da interface é muito significativa, e a natureza desta vem sendo utilizada para classificar os híbridos em diferentes classes.

\section{CLASSIFICAÇÃO}

Classe I: quando os componentes orgânicos e inorgânicos interagem através de pontes de hidrogênio, forças de van der Waals ou ligações iônicas. Materiais híbridos orgânico-inorgânicos da classe I podem ser preparados por diferentes rotas sintéticas:

a) componentes orgânicos (corantes, catalisadores, etc) podem ser imobilizados numa rede inorgânica, basicamente pela mistura homogênea destas moléculas, em solução, com precursores inorgânicos da rede, em um solvente comum. Durante o processo de policondensação do precursor inorgânico, as moléculas orgânicas ficam aleatoriamente dispersas, e no ponto de gel, ficam aprisionadas nos interstícios da rede inorgânica, permanecendo neles após a remoção do solvente, conforme ilustrado na Figura 1. Outra alternativa para se obter híbridos com esta concepção, é imergir um xerogel inorgânico em uma solução de moléculas orgânicas ${ }^{2}$. Após a remoção do solvente, estas moléculas também ficam confinadas na rede inorgânica.
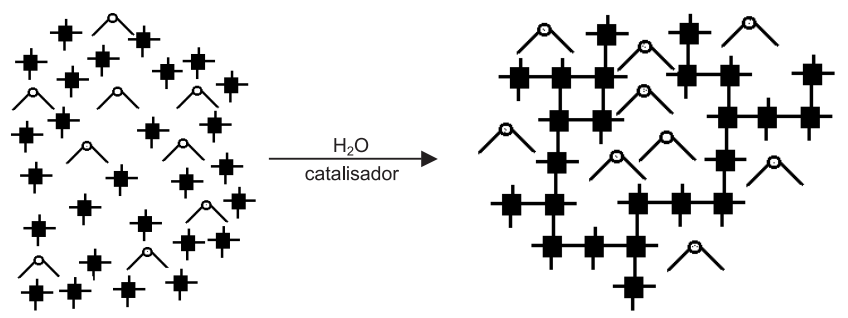

Figura 1. Moléculas orgânicas ( $)$ ) imobilizadas numa rede inorgânica (-十) preparada via sol-gel, a partir de precursores inorgânicos (-中)

Venton e Gudipati ${ }^{9}$ prepararam materiais da classe I, imobilizando as enzimas urease e invertase numa rede polimérica preparada a partir de uma mistura do tetraetoxissilano e 3-aminopropiltrietoxissilano, via processo sol-gel. A imobilização de catalisadores em redes poliméricas tem sido utilizada recentemente na busca de catalisadores com maior seletividade e estereoespecificidade ${ }^{10}$.

b) Monômeros orgânicos podem ser embebidos nos poros de uma matriz inorgânica e, então, polimerizados, em processos iniciados por radiação UV, por aquecimento ou por iniciadores de polimerização ${ }^{11}$. O polímero resultante fica entrelaçado à rede inorgânica, gerando uma rede polimérica semi-interpenetrante (semi-IPN) orgânico-inorgânica, conforme apresentado na Figura 2.

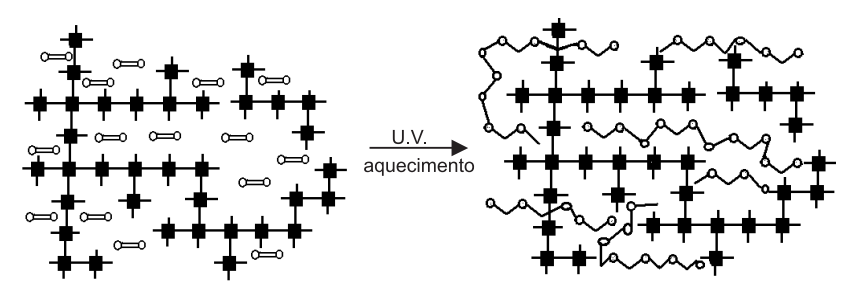

Figura 2. Monômeros orgânicos ( nica ( polimero (âa) c) Pela formação simultânea de duas redes independentes, a partir de precursores orgânicos e inorgânicos, adequadamente funcionalizados, sem ligação química entre as fases ${ }^{2}$, conforme mostra a Figura 3. Neste caso, o produto pode ser descrito como uma verdadeira rede polimérica interpenetrante (IPN). Um dos exemplos desta classe de híbridos, citado por Matejka e colaboradores $^{12}$, é uma IPN, onde o componente orgânico é a resina epoxi curada com amina polifuncional, e a fase inorgânica é constituída de sílica, preparada a partir da hidrólise e condensação do tetraetoxissilano, TEOS.

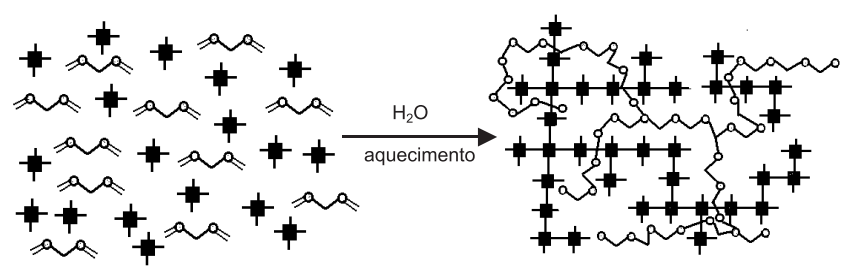

Figura 3. Formação simultânea de duas redes a partir de monômeros orgânicos ( $)$ precursores inorgânicos (

Classe II: Materiais híbridos orgânico-inorgânicos que são constituídos de estruturas nas quais os componentes orgânicos e inorgânicos são ligados de forma covalente ou iônico-covalente, conforme esquematizado na Figura 4.

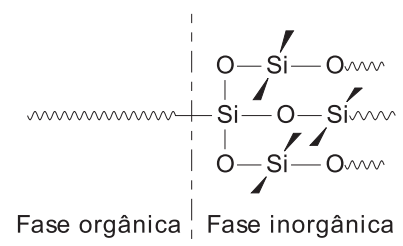

Figura 4. Material híbrido orgânico-inorgânico com ligações covalentes entre as fases

Ballard e colaboradores ${ }^{13}$ desenvolveram materiais híbridos orgânico-inorgânicos da classe II, utilizados em recobrimento de superfícies, a partir do óleo de soja como fase orgânica e diferentes misturas de óxidos de titânio e zircônio como fase inorgânica. As duas fases estão ligadas por fortes ligações covalentes. Características físicas como dureza, adesão, flexibilidade, resistência ao impacto e à fratura apresentaram efeito sinérgico.

Nas últimas duas décadas, o crescente interesse no desenvolvimento de novos materiais levou à preparação de silicatos organicamente modificados ORMOSILS ou "CERAMERS". Esses materiais podem ser sintetizados pela incorporação de polímeros orgânicos a redes de sílica gerada in situ ${ }^{14}$, através do processo sol-gel ${ }^{15}$. Materiais preparados a partir do TEOS e do poli(dimetilsiloxano), PDMS enquadram-se nesta classificação ${ }^{16}$.

Park e colaboradores ${ }^{16}$ investigaram uma série de géis de sílicatos organicamente modificados, com diferentes grupos alquilas, como metil, propil, fenil e octil, a fim de determinar o efeito desses grupos nas propriedades texturais, tais como área superficial, volume de poros, microporosidade e hidrofobicidade. Neste caso, observou-se que a presença dos grupos orgânicos promoveu uma redução na conectividade da rede de siloxano e na tensão superficial, causando um aumento na microporosidade.

Classe III: Baseada na combinação dos dois tipos de interação descritos nas classes I e II. Um exemplo deste híbrido é o material obtido por um polímero orgânico contendo grupos alcoxissilanos, 
$(\mathrm{SiOR})_{3}$, hidrolisáveis, e grupos aceptores de hidrogênio (carbonila, amina, imida, etc..), conforme esquematizado na Figura 5.

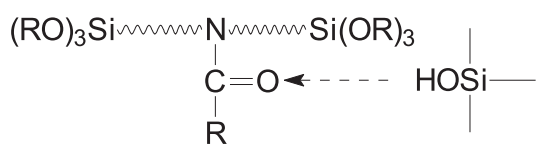

Figura 5. Material híbrido orgânico-inorgânico formado por ligações covalentes apresentando pontes de hidrogênio

\section{PREPARAÇÃO DE HÍBRIDOS ORGÂNICO-INORGÂNICOS}

\section{Processo sol-gel}

Independentemente da estratégia utilizada na preparação de um híbrido, o processo sol-gel é, indiscutivelmente, o mais empregado. $\mathrm{O}$ sol é constituído de uma suspensão de partículas coloidais (dimensão entre 1 e $1000 \mathrm{~nm}$ ) em um líquido ${ }^{17}$ e o gel é formado pela estrutura rígida de partículas coloidais (gel coloidal) ou por cadeias poliméricas (gel polimérico) que imobilizam a fase líquida nos seus interstícios ${ }^{1}$.

As reações químicas envolvidas num processo sol-gel convencional, baseado em derivados alcóxidos, são:

1 - Hidrólise do precursor ${ }^{17-19}$

$\mathrm{M}(\mathrm{OR})_{\mathrm{n}}+\mathrm{H}_{2} \mathrm{O} \longrightarrow \mathrm{M}(\mathrm{OH})(\mathrm{OR})_{\mathrm{n}-1}+\mathrm{ROH}$

onde $\mathrm{M}=\mathrm{Si}, \mathrm{Sn}, \mathrm{Ti}, \mathrm{Al}, \mathrm{Mo}$, etc

2 - A etapa posterior envolve reações de condensação que podem se processar por olação e oxolação ${ }^{18}$ :

$\begin{array}{ll}\text { olação: } & \mathrm{M}-\mathrm{OH}+\mathrm{H}_{2} \mathrm{O}^{+}-\mathrm{M} \longrightarrow \mathrm{M}-\mathrm{HO}^{+}-\mathrm{M}+\mathrm{H}_{2} \mathrm{O} \\ & \mathrm{M}-\mathrm{OH}+\mathrm{R}-\mathrm{HO}^{+}-\mathrm{M} \longrightarrow \mathrm{M}-\mathrm{HO}^{+}-\mathrm{M}+\mathrm{ROH}\end{array}$

oxolação: $\mathrm{M}-\mathrm{OH}+\mathrm{HO}-\mathrm{M} \longrightarrow \mathrm{M}-\mathrm{O}-\mathrm{M}+\mathrm{H}_{2} \mathrm{O}$

$\mathrm{M}-\mathrm{OH}+\mathrm{RO}-\mathrm{M} \longrightarrow \mathrm{M}-\mathrm{O}-\mathrm{M}+\mathrm{ROH}$

O processo sol-gel é relativamente complexo, envolvendo diversas variáveis, como tempo e temperatura da reação, natureza do catalisador, concentração de reagentes, etc. Estas variáveis determinam as características finais dos materiais, incluindo a porcentagem de hidrólise e condensação de grupos reativos, densidade de reticulação, homogeneidade do produto, etc. Além disso, aditivos químicos podem ser usados para melhorar o processo e obter materiais com melhores propriedades. Tais aditivos podem ser agentes estabilizantes ou agentes químicos controladores de secagem, DCCA, que em muitos casos permitem a obtenção do produto na forma de um monolito livre de trincas ${ }^{19,20}$.

A natureza do catalisador, ácido ou básico, influencia fortemente a cinética de reação, assim como a estrutura final do gel ${ }^{17,21}$. Se a hidrólise for catalisada por base, a reação deve se processar através de uma substituição nucleofílica, e a velocidade de hidrólise será maior que a velocidade de condensação, levando à formação de cadeias mais ramificadas no início do processo. Se a hidrólise for catalisada por ácido, vai ocorrer uma reação eletrofílica, e a velocidade da condensação será maior que a da hidrólise, levando à formação de cadeias mais longas e menos ramificadas no início do processo.

\section{Rotas de preparação}

Nesta parte, as principais rotas sintéticas para a preparação dos híbridos das classes I, II e III encontram-se descritas, além da química envolvida nesses processos. As rotas estão preparadas em função da natureza do constituinte inorgânico.

\section{Híbridos contendo sílica}

A hidrólise e condensação de alcóxidos de silício é um método relativamente barato e conveniente para a produção de silicatos, que podem ser finamente dispersos em diferentes matrizes poliméricas.

Esse processo ${ }^{1}$ pode ser descrito por reações de hidrólise e condensação ilustradas pelas Equações 1-3

$\equiv \mathrm{Si}-\mathrm{OR}+\mathrm{H}_{2} \mathrm{O} \rightarrow \mathrm{Si}-\mathrm{OH}+\mathrm{R}-\mathrm{OH}$ (hidrólise)

$\equiv \mathrm{Si}-\mathrm{OH}+\equiv \mathrm{Si}-\mathrm{OR} \rightarrow \equiv \mathrm{Si}-\mathrm{O}-\mathrm{Si} \equiv+\mathrm{R}-\mathrm{OH}$ (condensação)

$\equiv \mathrm{Si}-\mathrm{OH}+\equiv \mathrm{Si}-\mathrm{OH} \rightarrow \equiv \mathrm{Si}-\mathrm{O}-\mathrm{Si} \equiv+\mathrm{H}_{2} \mathrm{O}$ (condensação)

(onde $\mathrm{R}=$ metil, etil, iso-propil, propil)

As reações de hidrólise e condensação de alcoxissilanos requerem o uso de catalisadores. Diferentes estruturas são obtidas quando se utiliza catalisadores ácidos ou básicos ${ }^{17}$. Estes diferentes arranjos dos silicatos se refletem na distribuição dos domínios de sílica nos híbridos. Encontra-se bem estabelecido que os catalisadores ácidos produzem estruturas mais lineares e fáceis de dispersar que os catalisadores alcalinos ${ }^{22}$. Neste caso, estruturas mais ramificadas e condensadas são geradas, as quais não se dispersam bem em matrizes poliméricas. Em matrizes polares, como acrílicos ${ }^{23}$, poli(imidas) $)^{24}$, poli(amidas) $)^{25}$, poli(acetato de vinila) ${ }^{26}$ e poli(álcool vinílico) $)^{27}$, a presença de segmentos lineares ricos em grupos $\mathrm{Si}-\mathrm{OH}$ favorece a formação de pontes de hidrogênio entre os grupos carbonila e N-H destes polímeros, favorecendo a compatibilização entre as fases orgânica e inorgânica.

Mais recentemente, o uso de ácido polissilícico tem se mostrado conveniente quando se almeja um maior controle na estrutura molecular da sílica ${ }^{28}$. O ácido polissilícico é produzido pela adição de ácidos minerais a silicato de sódio, conforme ilustra a reação abaixo.

$\mathrm{Na}_{2} \mathrm{SiO}_{3}+2 \mathrm{H}^{+} \rightarrow$ " $\mathrm{H}_{2} \mathrm{SiO}_{3} "+2 \mathrm{Na}^{+}$

Conforme descrito para os alcóxidos, os grupos $\mathrm{Si}-\mathrm{OH}$ sofrem condensação, produzindo cadeias de silicato solúveis em solventes orgânicos. Por esse processo, podem ser produzidos híbridos de sílica tendo poli(metacrilato de 2-hidroxil etila) como matriz .

\section{Híbridos contendo siloxanos}

Analogamente ao descrito para híbridos/nanocompósitos, o termo silicato é, às vezes, confundido com o termo siloxano. A unidade $\mathrm{SiO}_{4 / 2}$ é o monômero que constitui a sílica, nesse caso o termo silicato é perfeitamente aplicável. Porém quando um silício apresenta-se ligado a um ou mais substituintes orgânicos e a um oxigênio, o termo siloxano é o mais apropriado. Entre os siloxanos, existe ainda uma sub-classe, a dos silsesquioxanos, onde um átomo de silício encontra-se ligado a um hidrogênio (ou um grupo orgânico) e a três oxigê$\operatorname{nios}^{29}$ (Figura 6).

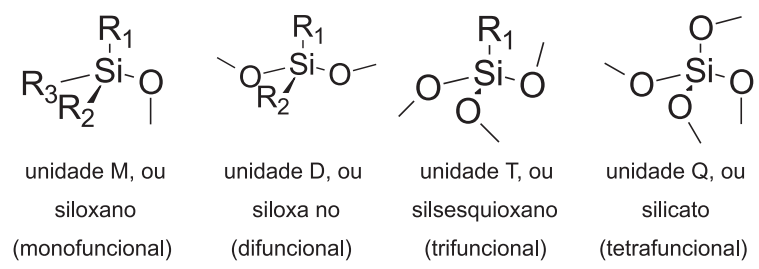

Figura 6. Fórmulas estruturais das unidades de repetição encontradas em polissiloxanos

A reatividade e compatiblidade dos siloxanos com matrizes poliméricas pode ser controlada não apenas pelas condições experi- 
mentais, mas também pelas características impostas pelo substituinte. Há uma infinidade de monômeros comercializados, contendo aminas, acrilatos, vinila, heterociclos ligados ao silício ${ }^{30}$. Existem duas estratégias básicas neste caso.

a. Preparação de polímeros com grupos alcóxidos. Dois ou mais monômeros reagem, dando origem a um co-polímero contendo grupos alcóxidos ao longo da cadeia. Por esse processo podem ser produzidos híbridos poli(metacrilato de metila)/siloxanos ${ }^{31}$, poli(imida)/siloxanos ${ }^{32}$, resinas epóxi modificadas com organossiloxanos $^{33}$, entre outros. Essa estratégia encontra-se ilustrada na Figura 7.<smiles>C=C(C)C(=O)OCCC[Si](OC)(OC)OC</smiles>
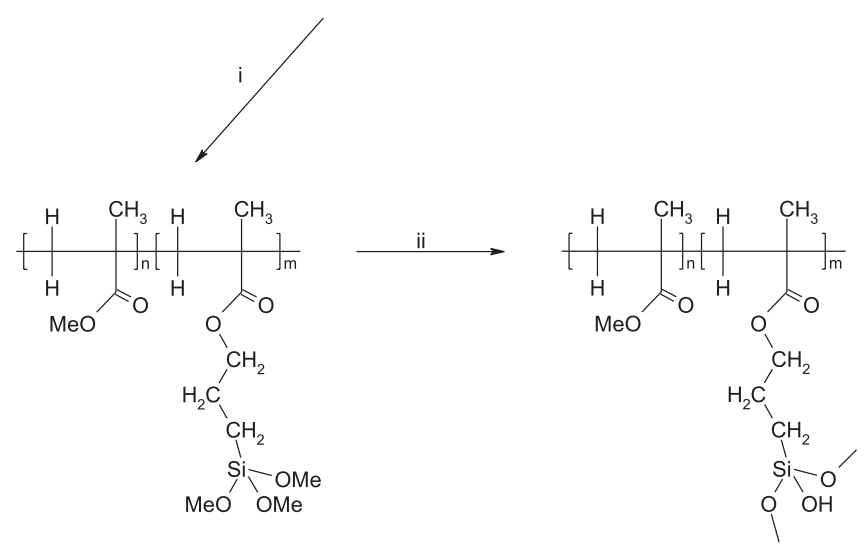

Figura 7. Rota para a produção de um híbdrido de poli(metacrilato de metila) e siloxanos: (i) co-polimerização do metacrilato de metila e do 3metacrilóxi-propiltrimetoxissilano (ii) policondensação dos grupos $\mathrm{Si}(\mathrm{OMe})_{3}$ dando origem a uma rede polimérica de siloxanos

b. Funcionalização de polímeros. Neste caso, um polímero é modificado quimicamente e os grupos alcóxidos ligados ao silício são introduzidos e, através de hidrólise e condensação, produzem os domínios de siloxanos. Por esse processo poli(sulfonas) ${ }^{34}$ e poli(éteres) contendo grupos aromáticos ${ }^{35}$ podem ser modificados. Polímeros sulfonados também podem ser funcionalizados por esse processo, utilizando-se organossiloxanos contendo grupos amino ${ }^{36}$. Exemplos encontram-se na Figura 8.

\section{Fosfatos organicamente modificados (fosfonatos) $)^{37}$}

Analogamente aos organossiloxanos, são moléculas contendo grupos orgânicos e unidades de ácido fosfônico. Graças à facilidade de funcionalização ${ }^{38}$, ácidos fosfônicos contendo grupos funcionais polimerizávies, tais como aminas, álcoois, insaturações, epóxidos, podem ser preparados por processos relativamente simples. Contudo, híbridos contendo fosfonatos não são tão comuns quanto os híbridos contendo organossiloxanos e silicatos.

\section{Óxidos de metais de transição e alumina}

Diferente do silício, alcóxidos de metais de transição e de alumínio (III) possuem elevada reatividade frente à hidrólise e condensação. Esse fato é justificável, levando-se em consideração a tendência desses metais em expandir o número de coordenação ${ }^{39}$. O Ti $\left(\mathrm{OC}_{2} \mathrm{H}_{5}\right)_{4}$ contém um átomo de titânio ligado a quatro grupos alcóxidos, po-

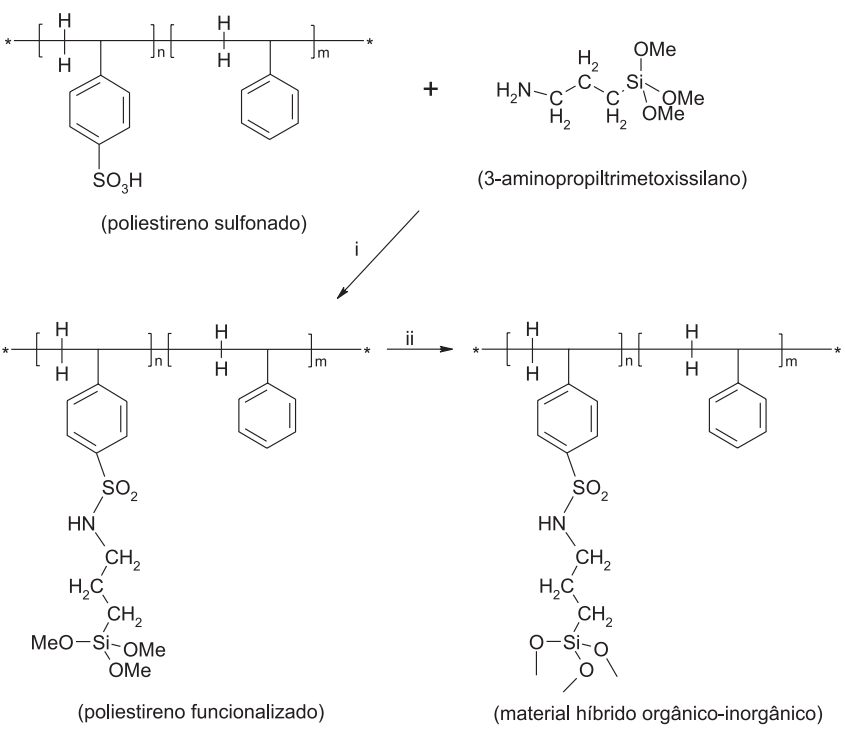

Figura 8. Rota para a produção de um híbdrido de poli(estireno) sulfonado e siloxanos: (i) funcionalização do polímero através de reação com 3-aminopropiltrimetoxissilano (ii) policondensação dos grupos - $\mathrm{Si}(\mathrm{OMe})_{3}$ dando origem a uma rede polimérica de siloxanos

rém a razão carga/raio iônico do cátion $\mathrm{Ti}^{+4}$ indica que o número de coordenação ideal para esse íon seria seis ${ }^{40}$. Deste modo, os alcóxidos de titânio formam oligômeros cujo tamanho e geometria são bastante afetados pelo tamanho do substituinte orgânico ligado ao oxigênio, quer por efeitos eletrônicos, quer por efeitos estéricos. $\mathrm{O}$ mesmo é válido para os alcóxidos de alumínio, estanho, zircônio, tungstênio, molibdênio e vanádio, entre outros ${ }^{39}$.

O processo de hidrólise e condensação desses alcóxidos é significativamente mais complexo que o descrito para as espécies $\mathrm{Si}(\mathrm{OR})_{4}$, em virtude do maior número de possibilidades estruturais, incluindo espécies com grupos - $\mathrm{OH}$ e -OR em ponte entre dois ou três íons metálicos.

A reatividade destes alcóxidos pode ser controlada pelo uso de agentes quelantes, que aumentam o número de coordenação do íon central, diminuindo a sua reatividade ${ }^{41}$. O ligante mais conhecido e utlizado é a 2,4-pentanodiona (acetil-acetona). Outros ligantes utlizados incluem ácido etanóico (ácido acético), amino-ácidos, ligantes bi- ou multidentados capazes de formar complexos relativamente estáveis com esses íons ${ }^{41}$.

Em muitos casos, o polímero já possui esses grupos, tais como os polímeros sulfonados, ou polímeros do ácido acrílico ou metacrílico. Deste modo, a formação de híbridos contendo esses óxidos finamente dispersos é possível.

No caso de polissiloxanos como matrizes poliméricas, existe ainda a possibilidade de formação de ligações M-O-Si, através de reações de condensação ${ }^{42}$. Por esse processo, poli(dimetilsiloxano) contendo grupos $-\mathrm{OH}$ no final de cadeia pode ser vulcanizado por alcóxidos de metais de transição.

\section{Materiais híbridos a partir de precursores com estrutura molecular controlada}

\section{Poliedros de silsesquioxanos (oligossilsesquioxanos)}

As unidades $\mathrm{RSiO}_{3 / 2}$ podem combinar-se formando poliedros contendo de 8 a 12 átomos de silício ${ }^{43}$. Embora as condições de preparo desses precursores em bons rendimentos nem sempre sejam simples, o uso destes tem se tornado cada vez mais comum, uma vez que o grupo $\mathrm{R}$ pode conter unidades capazes de participar de reações 
de polimerização ou de reagir com polímeros contendo grupos funcionais específicos ${ }^{44}$. Estruturas típicas desses poliedros podem ser vistas na Figura 9.
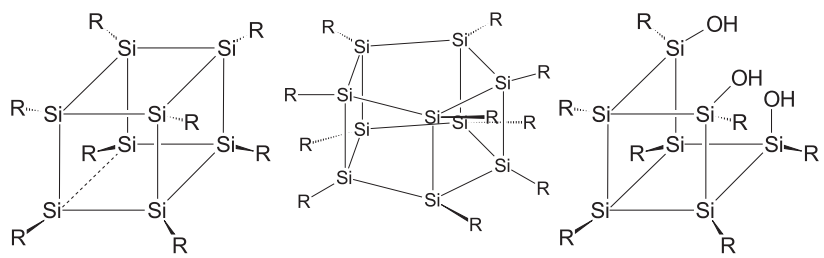

Figura 9. Modelos estruturais para poliedros de silsesquioxanos: (i) octâmero (esquerda) (ii) decâmero (centro) e (iii) estruturas parcialmente condensadas.(As arestas destes poliedros representam ligações Si-O-Si e R, um grupo orgânico)

A química destes poliedros tem-se desenvolvido com relativa rapidez, sendo possível também a preparação de poliedros contendo dois ou mais grupos funcionais diferentes, dando origem a materiais com diferentes estruturas moleculares e propriedades macroscópicas ${ }^{45}$.

\section{Poliedros a partir de silicatos}

Os silicatos também podem ser preparados na forma de poliedros de 8 a 12 átomos em rendimentos quantitativos ${ }^{46}$. Esses poliedros, por sua vez, podem reagir com organossilanos, dando origem a poliedros funcionalizados, com estruturas semelhantes às representada na Figura 9 onde $\mathrm{R}$, neste caso, corresponde a um grupo silil ($\mathrm{Si}\left(\mathrm{CH}_{3}\right)_{2} \mathrm{OH} ;-\mathrm{Si}\left(\mathrm{CH}_{3}\right)_{2} \mathrm{H} ;-\mathrm{Si}\left(\mathrm{CH}_{3}\right)_{2}\left(\mathrm{CH}=\mathrm{CH}_{2}\right)$, etc $)^{47}$.

\section{Polioxometalatos}

Recentemente, Schubert e colaboradores ${ }^{48}$ descreveram a preparação de espécies derivadas da reação entre alcóxidos de metais de transição e ácido acrílico/metacrílico, com um bom controle da estrutura molecular. Essas espécies são polioxometalatos, ou seja, espécies oligoméricas constituídas por metais de transição e oxigênio (semelhante ao dicromato e ao tricromato, porém muito mais estáveis) funcionalizadas com grupos acrílicos e metacrílicos, conforme ilustra a reação abaixo.

$$
\begin{array}{r}
6 \mathrm{Zr}(\mathrm{OR})_{4}+20 \mathrm{R}^{`} \mathrm{COOH} \rightarrow \mathrm{Zr}_{6}(\mathrm{OH})_{4} \mathrm{O}_{4}\left(\mathrm{OOCR}^{`}\right)_{12}+8 \mathrm{R}^{`} \mathrm{C}(\mathrm{O}) \mathrm{OR} \\
+16 \mathrm{ROH}
\end{array}
$$

A co-polimerização destes polioxometalatos com metacrilato de metila produz híbridos de poli(metacrilato de metila), PMMA, com óxidos com excepcional transparência e boa estabilidade térmica.

\section{Precursores derivados de heteropoliácidos ${ }^{49}$}

Heteropoliácidos constituem uma classe especial dos polioxometalatos. Normalmente um ânion central (fosfato, silicato, ou borato) encontra-se no centro de um poliedro, cujas arestas são compostas por óxidos de metais de transição. Os heteropoliácidos comerciais contém fósforo e molibdênio (ácido fosfomolíbdico, $\mathrm{H}_{3} \mathrm{PMo}_{12} \mathrm{O}_{40}$ ), fósforo e tungstênio (ácido fosfotúngstico, $\mathrm{H}_{3} \mathrm{PW}_{12} \mathrm{O}_{40}$ ), molibdênio e silício (ácido silicomolíbdico, $\mathrm{H}_{4} \mathrm{SiMo}_{12} \mathrm{O}_{40}$ ), tungstênio e silício (ácido silicotúngstico $\mathrm{H}_{4} \mathrm{SiW}_{12} \mathrm{O}_{40}$ ).

Estruturas onde um ou dois átomos de metal são substituídos por organossiloxanos (vide Figura 10), ou por fosfonatos já se encontram descritas na literatura ${ }^{49}$. No entanto, apenas um material híbrido foi preparado até então, contendo poli(estireno) e ácido silicotúngstico ${ }^{49}$.

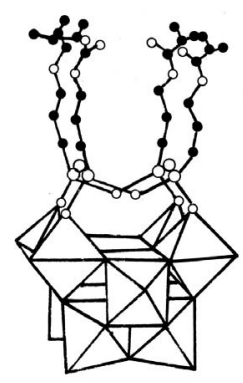

(i)

(ii) $\left[\mathrm{SiW}_{10} \mathrm{O}_{36}\right]^{8-}+4 \mathrm{RSi}\left(\mathrm{OCH}_{3}\right)_{3} \stackrel{\mathrm{H}_{2} \mathrm{O}}{\rightarrow}\left[\mathrm{SiW}_{10} \mathrm{O}_{36}\left(\mathrm{O}_{4} \mathrm{Si}_{4} \mathrm{R}_{4}\right)\right]^{4-}+8 \mathrm{CH}_{3} \mathrm{OH}+4 \mathrm{CH}_{3} \mathrm{O}^{-}$

Figura 10. (i) Estrutura do ácido silicotúngstico modificado com organossiloxanos (ii) Rota de síntese do precursor

\section{Aplicações}

Materiais híbridos orgânico-inorgânicos podem ser facilmente preparados e modificados com a grande variedade de combinações dos componentes disponíveis, conferindo diferentes contribuições nas propriedades do material resultante, o que possibilita modificações nas propriedades mecânicas, controle da porosidade e ajuste no balanço hidrofílico/hidrofóbico ${ }^{50}$. Essa grande versatilidade associada às suas propriedades ópticas, estabilidade química e termo-mecânica fazem com que esses materiais se apresentem com um grande potencial em diferentes aplicações, como no desenvolvimento de sensores químicos ${ }^{51}$, aplicações ópticas ${ }^{52}$, biomateriais ${ }^{53}$, em catálise ${ }^{54}$, revestimentos $^{55}$, aplicações cromatográficas ${ }^{56}$, preparação de membranas e materiais compósitos ${ }^{57}$.

\section{Sensores químicos}

O uso de materiais híbridos na fabricação de sensores químicos é devido, principalmente, à transparência óptica, estabilidade fotoquímica e eletroquímica destes materiais ${ }^{58}$. Os reagentes podem ser incorporados nos poros da matriz, por dopagem física, durante a gelificação $0^{59}$. Embora esse método seja bastante simples, pode ocorrer a lixiviação do dopante ${ }^{60}$. Os sensores podem ser preparados via polimerização de precursores organossilanos, contendo o reagente desejado, o qual é ligado covalentemente à estrutura do siloxano, impedindo assim sua saída da matriz. Através da síntese do vermelho de metila funcionalizado com grupos alcoxissilanos e copolimerização deste com tetrametoxissilano (TMOS), foi preparado um sensor de $\mathrm{pH}^{61}$. Materiais híbridos também são usados na fabricação de biosensores amperométricos, eletrodos indicadores e eletrodos potenciométricos. Esses eletrodos podem ser preparados pela adição de carbono em pó no ormosil sol e a pasta resultante é moldada em tubos de vidro onde é adaptado um contato elétrico ${ }^{62}$. Para a fabricação de um sensor amperométrico de glicose, foi sintetizado o alcóxido de ferrocenoacetamidassilano, através da reação do ácido ferrocenoacético com 3-aminopropiltrimetoxissilano ${ }^{63}$.

\section{Aplicações ópticas}

Esses materiais têm sido investigados para uso em dispositivos de óptica não linear (NLO), como guias de ondas ${ }^{64}$, etc. Materiais fotocromáticos são preparados pelo aprisionamento de corantes orgânicos, fotocromáticos, em matriz polimérica. Esses materiais 
fotocrômicos mudam de cor em resposta à absorção de luz e são usados principalmente em revestimento de proteção ${ }^{57}$. As primeiras tentativas na preparação de materiais NLO concentraram-se na imobilização, por dopagem física, dos grupos cromóforos por sol-gel. Uma desvantagem dessa técnica está associada ao movimento rotacional desses grupos nos poros da matriz, que permitem adquirir orientações randômicas, quando o campo elétrico é removido. A fim de superar essa limitação, foram preparados silicatos organicamente modificados, Ormosils, R-Si(OR') ${ }_{3}$, onde R = grupos cromóforos $^{65}$. Os grupos cromóforos foram covalentemente ancorados, restringindo, desse modo, sua mobilidade molecular.

\section{Biomateriais}

Materiais que podem substituir tecidos vivos, de forma apropriada, são denominados de biomateriais e devem apresentar propriedades físicas e biológicas compatíveis com esses tecidos hospedeiros, de modo a estimular uma resposta adequada dos mesmos. Tais propriedades caracterizam a biocompatibilidade ${ }^{66}$. Foi relatada a síntese e caracterização de materiais biocompatíveis e biodegradáveis à base de tetraetoxissilano (TEOS) e poli( $\varepsilon$-caprolactana), PCL, com potencial indicação no uso de implantes ósseos ${ }^{67}$. Híbridos orgânico-inorgânicos vêm sendo utilizados em aplicações odontológicas, na confecção de próteses e restaurações ${ }^{68}$.

Chang e colaboradoress ${ }^{69}$ testaram implantes de córneas artificiais em coelhos, preparadas por enxertia do poli(2-hidroxietilmetacrilato), pHEMA, em membranas de silicone, confirmando a biocompatibilidade do material.

\section{Catálise}

O desenvolvimento de novos materiais para catálise heterogênea vem sendo usado na preparação de suportes híbridos orgânicoinorgânicos, onde o catalisador é aplicado na superfície de diferentes suportes, como sílica, alumina, cloreto de magnésio e materiais zeólitos $^{57}$. A imobilização de catalisadores em membranas poliméricas tem sido uma das estratégias recentes utilizadas na busca de catalisadores heterogêneos, com maior seletividade, rendimento e velocidade para algumas reações ${ }^{54}$. Reatores de membranas catalíticas, que combinam simultaneamente as etapas de reação e separação, em uma única unidade de operação, podem representar uma alternativa em um grande número de reações industriais ${ }^{70}$.

\section{Revestimentos}

Os requisitos necessários para utilização de um material como revestimento incluem uma efetiva proteção contra corrosão, abrasão e que exiba boa adesão entre o revestimento e a superfície e, no caso de superfícies porosas, uma boa penetração nos poros do material ${ }^{71}$. Polímeros híbridos orgânico-inorgânicos vêm tendo um grande volume de aplicações como revestimento na indústria automotiva ${ }^{72}$. A maioria desses materiais, naturalmente, são protegidos por patentes $^{72}$. É condição necessária que a temperatura de cura do revestimento polimérico para aplicação em áreas grandes seja a ambiente, o que possibilita a aplicação "on-site" 73 . O processo sol-gel, mais uma vez, é o método mais adequado para esses casos, pois geralmente a cura ocorre à temperatura ambiente ${ }^{74}$.

Pilz e Römich ${ }^{73}$ sintetizaram materiais, via processo sol-gel, a partir do 3-glicidoxipropiltrimetoxissilano ou $\gamma$-metacriloxipropiltrimetossilano, modificados com diferentes silanos, condições de cura e aditivos. Esses híbridos foram testados como revestimentos de proteção contra corrosão em esculturas de bronze, apresentando boa adesão, estabilidade e reversibilidade (facilidade de remoção).

\section{Aplicações cromatográficas}

Materiais híbridos têm sido usados no desenvolvimento de fases estacionárias para cromatografia líquida ${ }^{75}$. Colunas para fase reversa ${ }^{76}$ ou fase normal podem ser preparadas pela escolha adequada dos precursores organossilanos. Seguindo uma nova tendência de combinar propriedades distintas em um único material, Silva et al. ${ }^{77}$ prepararam fases estacionárias reversas, contendo grupos polares do tipo uréia. Filmes para aplicação em cromatografia foram preparados através da hidrólise e condensação do metiltrimetoxissilano, na presença do vermelho de bromocresol, que funciona como catalisador e dopante formador de poros. O dopante é lavado da matriz com etanol, formando poros de tamanhos apropriados no filme ${ }^{78}$.

\section{Membranas}

A utilização de materiais híbridos tem recebido uma atenção especial na tecnologia de membranas, especialmente naquelas que atuam em processos de osmose reversa ${ }^{79}$, ultrafiltração ${ }^{80}$, diálise, troca de íons $^{79}$, pervaporação ${ }^{81}$, reatores catalíticos ${ }^{70}$, membranas condutoras de prótons usadas no desenvolvimento de células combustíveis $^{82}$, e membranas para separação de gases ${ }^{83}$. Diferentes materiais híbridos vêm sendo investigados na preparação de membranas para separação de gases, em vários grupos de pesquisa ${ }^{84}$. Raman e Brinker ${ }^{84}$ prepararam membranas com porosidade controlada a partir da hidrólise e co-condensação do metiltrietoxissilano com TEOS. As membranas exibiram boa permeabilidade frente aos gases $\mathrm{CO}_{2}, \mathrm{CH}_{4}$, $\mathrm{He}$ e $\mathrm{N}_{2}$ e foi obtida seletividade para o par $\mathrm{CO}_{2} / \mathrm{CH}_{4}$.

\section{Compósitos}

São materiais constituídos pela mistura de uma matriz (componente em maior quantidade) e cargas, que podem ser ativas, designadas como reforço e que são introduzidas para melhorar as propriedades térmicas e mecânicas do compósito, ou cargas inertes, que têm a finalidade de reduzir custos e facilitar a moldagem ${ }^{6}$. Tem sido descrito o emprego de carbeto de silício, sílica, óxido de alumínio e argilas como cargas ativas em compósitos de matriz polimérica, conferindo maior resistência mecânica e estabilidade térmica ${ }^{85}$. O processo sol-gel tem sido usado para preparar diferentes compósitos em matrizes de polímeros híbridos orgânico-inorgânicos ${ }^{86}$. Yoshida e colaboradores ${ }^{87}$ prepararam membranas à base de material compósito, obtidas a partir de uma matriz polimérica (híbrido orgânico-inorgânico) preparada com poli(dimetilsiloxano), PDMS, reticulado com núcleos dendríticos de pentaeritritoltriacrilato, aminoetilaminopropiltrimetoxissilano e tetraetoxissilano e como carga ativa foi usado o óxido de alumínio em diferentes proporções. A introdução do reforço levou a uma maior estabilidade térmica e acarretou um aumento nos valores do módulo de armazenamento (E'), proporcional à quantidade da carga.

\section{CONCLUSÕES}

A combinação de componentes inorgânicos e orgânicos ou bioativos, em escala nanométrica, em um único material levou ao desenvolvimento de uma nova área na ciência dos materiais. Materiais híbridos orgânico-inorgânicos vem se tornando uma atrativa área de estudos, devido a sua grande versatilidade e potencialidade, associada ao seu caráter multidisciplinar, que requer a associação de cientistas de diferentes domínios. A expectativa de expansão desta área do conhecimento é ilimitada, devido às infinitas possibilidades de combinações dos componentes e aplicações novas e sofisticadas surgirão desses estudos. 


\section{REFERENCIAS}

1. Hiratsuka, R. S.; Santilli, C. V.; Pulcinelli, S. H.; Quim. Nova 1995, 18, 171

2. Judeinstein, P.; Sanchez, C.; J. Mater. Chem. 1996, 6, 511.

3. Hu, Y. ; Chung, Y. J. ; Mackenzie, J. D.; J. Mater. Sci. 1993, 28, 6549.

4. Saegusa, T.; Pure Appl. Chem. 1995, 67, 1965.

5. Schubert, U.; Husing, N.; Lorenz, A.; Chem. Mater. 1995, 7, 2010.

6. Patterson, J. M.; Tsai, S. Em Encyclopedia of Polymer Science and Engineering; Mark, H. F.; Bikales, N. M.; Overbeyer, C. G.; Menges, G., eds.; John Wiley \& Sons Inc: New York, 1989, vol. 14; Shackelford, J. F.; Introduction to Materials Science for Engineers, 4a ed., Prentice Hall Inc: New Jersey, 1998, cap. 10; Matthews, F. L.; Rawlings, R. D.; Composite Materials: Enginnering and Science, Chapman \& Hall: London, 1996, cap. 1.

7. Roy, R.; Komarneni, S.; Roy, D. M.; Mater. Res. Soc. Symp. Proc. 1984, $32,347$.

8. Komarneni, S.; J. Mater. Chem. 1992, 2, 1219; Alexandre, M.; Dubois, P.; Mater. Sci. Eng., B 2000, 28, 1; Newnham, R. E.; Giniewicz, J. R. Em Comprehensive Composite Materials; Kelly, A.; Zweben, C.; Chou, T. W., eds.; Oxford, 2000, vol. 1, cap. 15.

9. Venton, D. L.; Gudipati, E.; Biochim. Biophys. Acta 1995, 1250, 117.

10. Adima, A.; Moreau, J. J. E.; Man, M. W. C.; Chirality 2000, 12, 411; Zhao, S. F.; Zhou, R. X.; Yang, Y. F.; Zheng, X. M.; React. Kinet. Catal. Lett. 2003, 79, 333; Yu, K. Q.; Jones, C. W.; Organometallics 2003, 22, 2571; Danelli, T.; Annunziata, R.; Benaglia, M.; Cinquini, M.; Cozzi, F.; Tocco, G.; Tetrahedron: Asymmetry 2003, 14, 461; Parton, R. F.; Vankelecom, I F. J.; Tas, D.; Janssen, K. B. M.; Gerrits, P. P. K.; Jacobs, P. A.; J. Mol. Catal. A: Chem. 1996, 113, 283; Helldörfer, M.; Alt, H. G.; Ebenhoch, J.; J. Appl. Polym. Sci. 2002, 86, 3021; Haag, M. C.; Dupont, J. Stedile, F. C.; Santos, J. H. Z.; J. Mol. Catal. A: Chem. 2003, 197, 223; Greco, P. P.; Stedile, F. C.; Santos, J. H. Z.; J. Mol. Catal. A: Chem. 2003, 197, 233.

11. Levy, D.; Einhorn, S.; Avnir, D.; J. Non-Cryst. Solids 1989, 113, 137; Pope, E. J. A.; Asami, A.; Mackenzie, J. D.; J. Mater. Res. 1989, 4, 1018.

12. Matejka, L.; Dušek, K.; Pleštil, J.; Krí•, J.; Lednický, F.; Polymer 1998, 40, 171.

13. Ballard, R. L.; Williams, J. P.; Njus, J. M.; Kiland, B. R.; Soucek, M. D.; Eur. Polym. J. 2001, 37, 381.

14. Mackenzie, J. D.; Huang, Q.; Iwamoto, T.; J. Sol-Gel Sci. Technol. 1996, 7,151 .

15. Ou, D. L.; Seddon, A. B.; J. Non-Cryst. Solids 1997, 210, 187; Yamada, N.; Yoshinaga, I.; Katayama, S.; J. Mater. Chem. 1997, 7, 1491.

16. Hu, Y.; Mackenzie, J. D.; J. Mater. Sci. 1992, 27, 4415; Park, M.; Komarneni, S.; J. Mater. Sci. 1998, 33, 3817; Guo, L.; Lee, J. H.; Beaucage, G.; J. Non-Cryst. Solids 1999, 243, 61.

17. Brinker, C. J.; Scherrer, G. W.; Sol-Gel Science, the Physics and Chemistry of Sol-Gel Processing, Academic: San Diego, 1990, cap. 2.

18. Fonseca, M. G.; Airoldi, C.; Quim. Nova 2003, 26, 699.

19. Hench, L. L.; West, J. K.; Chem. Rev. 1990, 90, 33.

20. Sanchez, C.; Livage, J. ; Henry, M.; Babonneau, F.; J. Non-Cryst. Solids 1988, 100,65 .

21. Sanchez, C.; Ribot, F.; New J. Chem. 1994, 18, 1007.

22. Schubert, U.; Hüsing, N.; Synthesis of Inorganic Materials, Wiley-VCH: Weinheim, Alemanha, 1a ed., 2001, cap. 4.

23. Berriot, J.; Montes, H.; Lequeux, F.; Long, D.; Sotta, P.; Europhys. Lett. 2003, 64, 50; Montes, H.; Lequeux, F.; Berriot, J.; Macromolecules 2003 38, 8107; Tong, X.; Tang, T.; Feng, Z. L.; Huang; B. T.; J. Appl. Polym. Sci. 2002, 86, 3532; Mizutani, R.; Oono, Y.; Matsuoka, J.; Nasu, H.; Kamiya, K.; J. Mater. Sci. 1994, 29, 5773.

24. Qiu, W. L.; Luo, Y. J.; Chen, F. T.; Duo, Y. Q.; Tan, H. M.; Polymer 2003, 43, 5821; Liu, J.; Gao, Y.; Wang, F. D.; Li, D. C.; Xu, J.; J. Mater. Sci. 2002 37, 3085; Ha, C. S.; Park, H. D.; Frank, C. W.; Chem. Mater. 2000, $12,839$.

25. Kim, J. H.; Lee, Y. M.; J. Membr. Sci. 2001, 193, 209; Ahmad, Z.; Sarwar, M. I.; Mark, J. E.; J. Appl. Polym. Sci. 1997, 63, 1345.

26. Donescu, D.; Zaharescu, M.; Gosa, K.; Moscu, S.; J. Dispersion. Sci. Technol. 2000, 21, 815; Beaudry, C. L.; Klein, L. C.; McCauley, R. A.; J. Thermal. Anal. 1996, 46, 55.

27. Uragami, T.; Okazaki, K.; Matsugi, H.; Miyata, T.; Macromolecules 2002, 35, 9156; Pereira, A. P. V.; Vasconcelos, W. L.; Orefice, R. L.; J. Non-Cryst. Solids 2000, 273, 180.

28. Hsu, Y. G.; Lin, K. H.; Chiang, I. L.; Mater. Sci. Eng., B. 2001, 87, 31; Hajji, P.; David, L.; Gerard, J. F.; Pascault, J. P.; Vigier, G.; J. Polym. Sci., Part B: Polym. Phys. 1999, 37, 3172; Rueda-Rodriguez, C.; Arias-Garcia, C.; Fortune, R.; Bardet, L.; Eur. Polym. J. 1998, 34, 645.

29. Prado, L. A. S. de A.; Tese de Doutorado, Universidade Estadual de Campinas, Brasil, 2001; Baney, R. H.; Itoh, M.; Sakakibara, A.; Suzuki, T.; Chem. Rev. 1995, 95, 1049; Loy, D. A.; Shea, K. J.; Chem. Rev. 1995, 95,1431
30. http://www.abcr.de, acessada em Julho 2004; http://www.sigmaaldrich.com, acessada em Julho 2004.

31. Sarmento, V. H. V.; Dahmouche, K.; Santilli, C. V.; Pulcinelli, S. H.; Craievich, A. F.; J. Appl. Crystallogr. 2003, 36, 473; Kotoky, T.; Ray, B. C.; Dolui, S. K.; J. Polym. Mater. 2003, 20, 257; Gunji, T.; Okonogi, H.; Sakan, T.; Takamura, N.; Arimitsu, K.; Abe, Y.; Appl. Organomet. Chem. 2003, 17, 580; Costa, R. O. R.; Lameiras, F. S.; Vasconcelos, W. L.; J. SolGel Sci. Technol. 2003, 27, 343; Jang, J.; Park, H.; J. Appl. Polym. Sci. 2002, 85, 2074; Costa, R. O. R.; Vasconcelos, W. L.; J. Non-Cryst. Solids 2002, 304, 84; Saito, R.; Kuwano, K.; Tob, T.; J. Macromol. Sci., Pure Appl. Chem. 2002, 39, 171; Tissot, I.; Reymond, J. P.; Lefebvre, F.; BourgeatLami, E.; Chem. Mater. 2002, 14, 1325; Chan, C. K.; Chu, I. M.; Lee, W.; Chin W. K.; Macromol. Phys. Chem. 2001, 202, 911; Jang, J.; Bae, J.; Kang, D.; J. Appl. Polym. Sci. 2001, 82, 2310; Tai, H.; Sergienko, A.; Silverstein, M. S.; Polymer 2001, 42, 4473; Jana, R. N.; Bhunia, H. P. Nando, G. B.; Termochim. Acta 1997, 302, 1; Gilmer, T. C.; Hall, P. K.; Ehrenfeld, H.; Wilson, K.; Bivens, T.; Clay, D.; Endreszl, C.; J. Polym. Sci., Part A: Polym. Chem. 1996, 34, 1025.

32. Park, H. B.; Kim, J. K.; Nam, S. Y.; Lee, Y. M.; J. Membr. Sci. 2003, 220 , 59; Wang, H. M.; Tao, X. M.; Newton, E.; High Perform. Polym. 2002, 14, 271; Park, H. B.; Kim, J. H.; Kim, J. K.; Lee, Y. M.; Macromol. Rapid Commun. 2002, 23, 544; Sysel, P.; Hobzova, R.; Sindelar, V.; Brus, J.; Polymer 2001, 42, 10079; Andre, S.; Guida-Pietrasanta, F.; Rousseau, A.; Boutevin, B.; J. Polym. Sci., Part A: Polym. Chem. 2001, 39, 2414; Tsai, M. H.; Whang, W. T.; J. Appl. Polym. Sci. 2001, 81, 2500; Furukawa, N.; Yuasa, M.; Kimura, Y.; Polymer 1999, 40, 1853; McGrath, J. E.; Dunson, D. L.; Mecham, S. J.; Hedrick, J. L.; Adv. Polym. Sci. 1999, 140, 61; Furukawa, N.; Yuasa, M.; Yamada, Y.; Kimura, Y.; Polymer 1998, 39, 2941.

33. Kumar, S.A.; Narayanan, T. S. N. S.; Prog. Org. Coat. 2002, 45, 323; Chiang, C. L.; Ma, C. C. M.; Eur. Polym. J. 2002, 38, 2219; Kumar, S. A.; Alagar, M.; Mohan, V.; J. Mater. Eng. Perform. 2002, 11, 123; Ochi, M.; Takahashi, R.; Terauchi, A.; Polymer 2001, 42, 5151; Lin; Y. H.; Pramoda; K. P.; He, C. B.; Chen, W. Y.; Chung, T. S.; J. Polym. Sci., Part A: Polym. Chem. 2001 39, 2215; Canto, C. F.; Radovanovic, E.; Yoshida, I. V. P.; Acta Microscopica, 2000 9, 315; Canto, C. A. F.; Prado, L. A. S. de A.; Yoshida, I. V. P.; Resumo do $5^{\circ}$ Congresso Brasileiro de Ciência e Engenharia de Materiais, Águas de São Pedro, Brasil, 2000; Matejka, L.; Dukh, O.; Kolarik, J.; Polymer 2000, 41, 1449.

34. Kim, S. H.; Woo, H. G.; Kim, J. S.; Lee, H. W.; Kim, W. G.; J. Polym. Sci., Part A: Polym. Chem. 2001, 39, 2937.

35. Noel, J. L.W.; Wilkes, G. L.; Mohannty, D. K.; McGrathy, D. E.; J. Appl. Polym. Sci. 1990, 40, 1177.

36. Nunes, S. P.; Ruffmann, B.; Rikowski, E.; Vetter, S.; Richau, K.; J. Membr. Sci. 2002, 203, 215

37. Mutin, P. H.; Guerrero, G.; Vioux, A.; C. R. Chim. 2003, 6, 1153; Mao, J. G.; Wang, Z. K.; Clearfield, A.; Inorg. Chem. 2002, 41, 6106; Clearfield, A.; Sharma, C. V. K.; Zhang, B. P.; Chem. Mater. 2001, 13, 3099.

38. Cadogan, J. I. G. Em Advances in Radical Chemistry; Willians, G. H., ed.; Academic Press: London, 1990, cap. 5; Page, P.; Blonski, C.; Périé; J. Bioorg. Med. Chem. 1999, 7, 1403; Pelaprat, N.; Brondino, C.; Rigal, G.; Boutevin, B.; Eur. Polym. J. 1996, 32, 761; Campbell, I. G. M.; Raza, S. M.; J. Chem. Soc. (C) 1971, 1836.

39. Sanchez, C.; Ribot, F.; New J. Chem. 1994, 18, 1007.

40. Greenwood, N. N.; Earnshaw, A.; Chemistry of the Elements, ButterworthHeinemann: Oxford, Inglaterra, 2a ed., 1997, cap. 21.

41. Gbureck, U.; Probst, J.; Thull, R.; J. Sol-Gel Sci.Technol. 2003, 27, 157; Hubert-Pfalzgraf, L. G.; Coord. Chem. Rev. 1998, 178, 967; Schubert, U.; Tewinkel, S.; Lamber, R.; Chem. Mater. 1996, 8, 2047; Rude, E.; Llorens, J.; Mans, C.; Colloids Surf., A 1996, 119, 57.

42. Julian, B.; Gervais, C.; Cordoncillo, E.; Escribano, P.; Babonneau, F.; Sanchez, C.; Chem. Mater. 2003, 15, 3026; Vu, B. T. N.; Mark, J. E.; Schaefer, D. W.; Compos. Inter. 2003, 10, 451; Rajan, G. S.; Sur, G. S.; Mark, J. E.; Schaefer, D. W.; Beaucage, G.; J. Polym. Sci., Part B: Polym. Phys. 2003, 41, 1897; Kawamura, T.; Urayama, K.; Kohjiya, S.; J. Chem. Phys. 2000, 112, 9105; Yamada, N.; Yoshinaga, I.; Katayama, S.; J. Mater. Res. 1999, 14, 1720; Breiner, J. M.; Mark, J. E.; Beaucage, G.; J. Polym. Sci., Part B: Polym. Phys. 1999, 37, 142; Hild, G.; Prog. Polym. Sci. 1998, 23, 1019.

43. Bassindale, A. R.; Liu, Z. H.; MacKinnon, I. A.; Taylor, P. G.; Yang, Y. X.; Light, M. E.; Horton, P. N.; Hursthouse, M. B.; J. Chem. Soc., Dalton Trans. 2003, 2945.

44. Huang, J. C.; He, C. B.; Xiao, Y.; Mya, K. Y.; Dai, J.; Siow, Y. P.; Polymer 2003, 44, 4491; Richter, I.; Burschka, C.; Tacke, R.; J. Organomet. Chem. 2002, 646, 200; Ropartz, L.; Foster, D. F.; Morris, R. E.; Slawin, A. M. Z.; Cole-Hamilton, D. J.; J. Chem. Soc., Dalton Trans. 2002, 1997; Haddad, T. S.; Viers, B. D.; Phillips, S. H.; Inorg. Organomet. Polym. 2001, 11, 155. 
45. Bassindale, A. R.; Parker, D. J.; Taylor, P. G.; Watt, A. C.; Can. J. Chem. 2003, 81, 1341; Duchateau, R.; Chem. Rev. 2002, 102, 3525; Lorenz, V.; Spoida, M.; Fischer, A.; Edelmann, F. T.; J. Organomet. Chem. 2001, 625, 1; Feher, F. J.; Wyndham, K. D.; Soulivong, D.; Nguyen, F.; J. Chem. Soc., Dalton Trans. 1999, 1491.

46. Ray, N.; Plaisted, R.; J. Chem. Soc., Dalton Trans. 1983, 475.

47. Hasegawa, I.; Ino, K.; Ohnishi, H. Appl. Organomet. Chem. 2003, 17, 287; Choi, J.; Yee, A. F.; Laine, R. M.; Macromolecules 2003, 36, 5666; Costa, R. O. R.; Vasconcelos, W. L.; Tamaki, R.; Laine, R. M.; Macromolecules 2001, 34, 5398 .

48. Schubert, U.; J. Sol-Gel Sci. Technol. 2003, 26, 47; Gross, S.; Di Noto, V.; Schubert, U.; J. Non-Cryst. Solids 2003, 322, 154; Kickelbick, G.; Holzinger; D.; Brick, C.; Trimmel, G.; Moons, E.; Chem. Mater. 2002, 14 , 4382; Trimmel, G.; Moraru, B.; Gross, S.; Di Noto, V.; Schubert, U.; Macromol. Symp. 2001, 175, 357; Schubert, U.; Chem. Mater. 2001, 13, 3487; Trimmel, G.; Gross, S.; Kickelbick, G.; Schubert, U.; Appl. Organomet. Chem. 2001, 15, 401.

49. Wang, X.; Liu, J.; Li, J.; Liu J.; Inorg. Chem. Commun. 2001, 4, 372; Mazeaud, A.; Dromze, Y.; Thouvenot, R.; Inorg. Chem. 2000, 39, 4735; Mayer, C. R.; Fournier, I.; Thouvenot, R.; Chem. Eur. J. 2000, 6, 105; Judeinstein, P.; Chem. Mater. 1991, 4, 4.

50. Zoppi, R. A.; Castro, C. R.; Yoshida, I. V. P.; Nunes, S. P.; Polymer 1997, $38,5705$.

51. Levy, D.; New J. Chem. 1994, 18, 1073; He, H.; Fraatz, R. J.; Leiner, M. J. P.; Rehn, M. M.; Tusa, J. K.; Sens. Actuators, B 1995, 29, 246; Tsujimura, Y.; Yokoyama, M.; Kimura, K.; Anal. Chem. 1995, 67, 2401; Livage, J.; Curr. Opin. Solid State Mater. Sci. 1997, 2, 132; Calvo-Munoz, M. L.; Truong, T. T.; Tran-Thi, T. H.; Sens. Actuators, B 2002, 87, 173; CalvoMunoz, M. L.; Roux, C.; Brunet, F.; Bourgoin, J. P.; Ayral, A.; El-Mansouri, A.; Tran-Thi, T. H.; J. Mater. Chem. 2002, 12, 461; de Campos, J. D. R.; Buffon, R.; New J. Chem. 2003, 27, 446.

52. Sanchez, C.; Lebeau, B.; Ribot, F.; In, M.; J. Sol-Gel Sci. Technol. 2000, 19, 31; Krug, H.; Schmidt, H.; New J. Chem. 1994, 18, 1125; CroutxeBarghorn, C.; Soppera, A.; Chevallier, M.; Macromol. Mater. Eng. 2003, 288, 219; Sanz, N.; Baldeck, P. L.; Ibanez, A.; Synth. Met. 2000, 115, 229; Zaccaro, J.; Baruchel, J.; Ibanez, A.; J. Mater. Chem. 1999, 9, 403; Boilot, J. P.; Biteau, J.; Chaput, F.; Gacoin, T.; Brun, A.; Darracq, B.; Georges, P.; Levy, Y.; Pure Appl. Opt. 1998, 7, 169.

53. Zoppi, R. A.; Contant, S.; Duek, E. A. R.; Marques, F. R.; Wada, M. L. F.; Nunes, S. P.; Polymer 1999, 40, 3275; Lee, S. D.; Hsiue, G. H.; Kao, C. Y.; Chang, T.; Biomaterials 1996, 17, 587.

54. Moreau, J. J. E.; Man, M. W. C.; Coord. Chem. Rev. 1998, 178, 1073; Farrusseng, D.; Julbe, A.; Lopez, M.; Guizard, C.; Catal. Today 2000, 56, 211; Clark, J. H.; Macquarrie, D. J.; Chem. Commun. 1998, 8, 853; Villemin, D.; Jaffres, P. A.; Nechab, B.; Courivaud, F.; Tetrahedron Lett. 1997, 38, 6581; Epifani, M.; Leo, G.; Lomascolo, M.; Vasanelli, L.; Manna, L.; J. Sol-Gel Sci. Technol. 2003, 26, 441; Teixeira, S.; Dallmann, K.; Schuchardt, U.; Buffon, R.; J. Mol. Catal. A: Chem. 2002, 182, 167.

55. Mackenzie, J. D.; Bescher, E. P.; J. Sol-Gel Sci. Technol. 1998, 13, 371; Kasemann, R.; Schmidt, H.; New J. Chem. 1994, 18, 1117.

56. Cheng, Y. F.; Walter, T. H.; Lu, Z. L.; Iraneta, P.; Alden, B. A.; Gendreau, C.; Neue, U. D.; Grassi, J. M.; Carmody, J. L.; O’Gara, J. E.; Fisk, R. P.; LCGC North Am. 2000, 18, 1162.

57. Collinson, M. M.; Microchim. Acta 1998, 129, 149

58. Avnir, D.; Acc. Chem. Res. 1995, 28, 328; Lev, O.; Tsionsky, M.; Rabinovich, L.; Glezer, V.; Sampath, S.; Pankratov, I.; Gun, J.; Anal. Chem. 1995, 67, 22A

59. Avnir, D.; Levy, D.; Reisfeld, R.; J. Phys. Chem. 1984, 88, 5956.

60. Severin-Vantilt, M. M. E.; Oomen, E. W. J. L.; J. Non-Cryst. Solids 1993, 6,38 .
61. Aharonson, N.; Altstein, M.; Avidan, G.; Avnir, D.; Bronshtein, A.; Lewis, A.; Liberman, K.; Ottolenghi, M.; Polevaya, Y.; Rottman, C.; Samuel, J.; Shalom, S.; Strinkovski, A.; Turniansky, A.; Mater. Res. Soc. Symp. Proc. 1994, 346, 519

62. Sampath, S.; Lev, O.; Anal. Chem. 1996, 68, 2015

63. Lev, O.; Gun, J.; Anal. Chim. Acta 1996, 336, 95.

64. Burland, D. M.; Miller, R. D.; Walsh, C. A.; Chem. Rev. 1994, 94, 31.

65. Sanchez, C.; Lebeau, B.; Pure Appl. Opt. 1996, 5, 689; Lebeau, B.; Brasselet, S.; Zyss, J. Sanchez, C.; Chem. Mater. 1997, 9, 1012.

66. Kawachi, E. Y.; Bertran, C. A.; Reis, R. R.; Alves, O. L.; Quim. Nova 2000, 23,518 .

67. Tian, D.; Blacher, S.; Dubois, Ph.; Jérôme, R.; Polymer 1998, 39, 855; Tian, D.; Blacher, S.; Jerome, R.; Polymer 1999, 40, 951.

68. Jagger, D. C.; Harrison, A.; Jandt, K. D.; J. Oral Rehabilitation 1999, 26, 185; Skrtic, D.; Antonucci, J. M.; Eanes, E. D.; Eldelman, N.; Biomaterials 2004, 25, 1141 .

69. Lee, S. D.; Hsiue, G. H.; Kao, C. Y.; Chang, P. C. T.; Biomaterials 1996, 17, 587.

70. Rangel, M. C.; Quim. Nova 1997, 20, 486.

71. Kasemann, R.; Schmidt, H.; New J. Chem. 1994, 18, 1117.

72. Arkles, B.; MRS Bull 2001, may, 402.

73. Pilz, M.; Römich, H.; J. Sol-Gel Sci. Technol. 1997, 8, 1075.

74. Airoldi, C.; Farias, R. F. de; Quim. Nova 2004, 27, 84

75. Neue, U. D.; Walter, T. H.; Alden, B. A.; Jiang, Z. P.; Fisk, R. P.; Cook, J. T.; Glose, K. H.; Carmody, J. L.; Grassi, J. M.; Cheng, Y. F.; Lu, Z. L.; Crowley, R. J.; American Laboratory 1999, 31, 36.

76. Silva, C. R.; Jardim, I. C. S.; Collins, C. H.; Airoldi, C.; Quim. Nova 2004, 27,270

77. Silva, C. R.; Jardim, I. C. S.; Collins, C. H.; Airoldi, C.; J. Chromatogr., A 2003, 987, 139; Silva, C. R.; Jardim, I. C. S.; Collins, C. H.; Airoldi, C.; J. Chromatogr., A 2003, 987, 127.

78. Tsionsky, M.; Vanger, A.; Lev, O.; J. Sol-Gel Sci. Technol. 1994, 2, 595.

79. Maldonado, J.; Membranas e Processos de Separação, Instituto Nacional de Tecnologia, INT: Rio de Janeiro, 1991.

80. Laue, T. M. Em ref. 6; vol. 17.

81. Sforça, M. L.; Yoshida, I. V. P.; Borges, C. P.; Nunes, S. P.; J. Appl. Polym. Sci. 2001, 82, 178.

82. Ponce, M. L.; Prado, L.A. S. de A.; Silva, V.; Nunes, S. P.; Desalination 2004, 162, 383; Ponce, M. L.; Prado, L.; Ruffmann, B.; Richau, K.; Mohr, R.; Nunes, S. P.; J. Membr. Sci. 2003, 217, 5; Prado, L. A. S. de A.; Wittich, H.; Schulte, K.; Goerigk, G.; Garamus, V. M.; Willumeit, R.; Ruffmann, B.; Nunes, S. P.; J. Polym. Sci., Part B: Polym. Phys. 2004, 42, 567; Ruffmann, B.; Silva, H.; Schulte, B.; Nunes, S. P.; Solid State Ionics 2003, 162-163, 269; Honma, I.; Nomura, S.; Nakajima, H.; J. Membr. Sci. 2001, 185, 83.

83. Cot, L.; Ayral, A.; Durand, J.; Guizard, C.; Hovnanian, N.; Julbe, A.; Larbot, A.; Solid State Sci. 2000, 2, 313; He, Y.; Yang, J.; Li, H.; Huang, P.; Polymer 1998, 39, 3393; Pandey, P.; Chauhan, R. S.; Prog. Polym. Sci. 2001, 26, 853.

84. Smaihi, M.; Jermoumi, T.; Marignan, J.; Noble, R. D.; J. Membr. Sci. 1996, 116, 211; Guizard, C.; Lacan, P.; New J. Chem. 1994, 18, 1097; Raman, N. K.; Brinker, C. J.; J. Membr. Sci. 1995, 105, 273; Sforça, M. L.; Yoshida, I. V. P.; Borges, C. P.; Nunes, S. P.; J. Appl. Polym. Sci. 2001, 82, 178; Zoppi, R. A.; Yoshida, I. V. P.; Nunes, S. P.; Polymer 1998, 39, 1309.

85. Kusakabe, K.; Li, Z. Y.; Maeda, H.; Morooka, S.; J. Membr. Sci. 1995, 103, 175; Li, Z. Y.; Kusakabe, K.; Morooka, S.; J. Membr. Sci. 1996, 118, 159.

86. Ahmad, Z.; Sarwar, M. I.; Polymer 1997, 38, 4523; Ochi, M.; Takahashi, R.; Terauchi, A.; Polymer 2001, 42, 5151; Hu, Q.; Marand, E.; Polymer 1999, 40, 4833; Nunes, S. P.; Peinemann, K. V.; Ohlrogge, K.; Alpers, A.; Keller, M.; Pires, A. T. N.; J. Membr. Sci. 1999, 157, 219.

87. José, N. M.; Redondo, S. U. A.; Yoshida, I. V. P.; Anais do XV Congresso Brasileiro de Engenharia e Ciências dos Materiais, Natal, Brasil, 2002. 